\title{
A Rapidly Progressive Hydatid Disease Imitating Metastatic Malignancy: An Unusual Multi-Organ Presentation. Report of a Case and Review of Literature
}

\author{
Qays A. Hassan Al-Timimy* \\ Section of Radiology, Department of Surgery, Al-kindy College of Medicine, University of Baghdad, Baghdad, Iraq
}

${ }^{*}$ Corresponding author: Qays A. Hassan Al-Timimy, Section of Radiology, Department of Surgery, Al-kindy College of Medicine, University of Baghdad, Baghdad, Iraq, Tel: 9647722604163, E-mail: qtimeme@yahoo.com

Citation: Qays A. Hassan Al-Timimy (2016) A Rapidly Progressive Hydatid Disease Imitating Metastatic Malignancy: An Unusual Multi-Organ Presentation. Report of a Case and Review of Literature. SAJ Cas Rep 3: 104

Article history: Received: 19 November 2016, Accepted: 12 December 2016, Published: 15 December 2016

\begin{abstract}
Hydatid disease is still a major health and socio-economic problem in endemic regions. It is the most frequent parasitic infection that commonly affects the liver and rarely involves multiple organs. Herein, I report an extraordinary case of multi-organ hydatid disease that presented with rapidly progressive disseminated nature that mimic metastatic malignancy highlighting the interest of keeping a high level of clinical suspicion of this diagnosis especially in endemic regions.
\end{abstract}

Keywords: Hydatid Cyst; Multi-organ; Case report; Parasitic Diseases

\section{Introduction}

Hydatidosis is an endemic zoonotic infection caused by the parasitic tapeworm Echinococcus granulosus (E.granulosus) that infects herbivores and humans in its larvae stage. Middle East, and thus Iraq, is considered a highly endemic area in which the infection can cause serious public health problems. The disease commonly affects the liver (75\%), and less frequently the lungs (15\%) followed by other regions of the body such as the spleen, kidney, orbit, heart, brain and bone (10\%) [1]. Recently, it has been noticed that the number of cases with multi-organ localizations and multiple cysts has increased. Abdominal echinococcosis mostly hepatic cysts accompanying pulmonary cysts constitute most multi-organ localizations. There are only a few articles in literature dealing separately with such cases [2]. Disseminated Multi-organ hydatidosis is a serious potentially fatal infrequent form that usually results from the rupture of a one cyst, with subsequent seeding of protoscolices in other organs [3]. Here, I describe the case of a 22-year-old female with multi-organ hydatid disease and review of the relevant literature. The particularity of this case is related to its unusual clinical presentation that manifested by rapidly progressive nature. The purpose of this paper is to emphasize the fact that hydatid disease can involve any organ of the body and can have an unusual presentation resembling metastatic malignancy; therefore, a high index of clinical suspicion and awareness is necessary for correct diagnosis and avoiding diagnostic pitfalls and unnecessary investigations, which can have major consequences and complications for patients.

\section{Case Report}

A 22-year-old Iraqi female complaining of dyspnea and cough, for previous 6 months, treated periodically by a general practitioner with some antibiotics and steroid drugs which helped to alleviate her symptoms; however, she continued to experience intermittent cough. Recently, 2 weeks before the admission to my hospital, she suffered from repeated episodes of intense cough, followed by vomiting of colorless fluid and white grape skin-like membranes. She reports no significant medical history outside an appendectomy at age 12 and when she was 20 years old, she experienced acute abdominal pain and, at that time, an abdominal ultrasound was absolutely normal and she was treated symptomatically. There was no history of weight loss, change in bowel habit, or urinary symptoms. Clinical and physical examinations revealed apyrexia, normal blood pressure and heart rate, coarse crackles at middle and lower area of both lungs and mild tenderness in the right hypochondrium with hepatomegaly. Her laboratory tests showed leukocytosis with eosinophilia (leukocytes, 14,500/ $\mathrm{mm}^{3}$; eosinophils, 30\%), and mild elevation of both the C-reactive protein level and the erythrocyte sedimentation rate.CXR showed multiple well defined rounded radio opacities scattered throughout both lungs imitating metastatic malignancy of lung (Figure 1). Abdominal ultrasound records multiple thin wall cystic lesions of varying sizes involving diffusely the hepatic parenchyma as well as three cysts seen in the spleen (Figure 2). Abdominal and chest computed tomography (CT) scan was performed and showed multiple thin wall cystic masses with homogeneous fluid density 
contains in the liver, lungs and spleen (Figure 3). Some cysts show detached membrane and daughter vesicles within. These imaging findings were generally sufficient for diagnosis of hydatid disease. Although, the patient reports no contact with sheep or dogs at home or at work. She was university student and lives in an urban area in Baghdad with her family. After imaging procedures, she was hospitalized. Serologic test results for echinococcus by means of an indirect haemagglutination test were positive. Then, the diagnosis of hydatid disease was confirmed, and chemotherapy was decided. My patient was treated with oral albendazole (400 mg twice daily) in association with praziquentel $50 \mathrm{mg} / \mathrm{kg}$ per day during 15 days. She was discharged after two weeks hospital stay with a good general health state and she will be maintained on albendazole with regular follow-up.

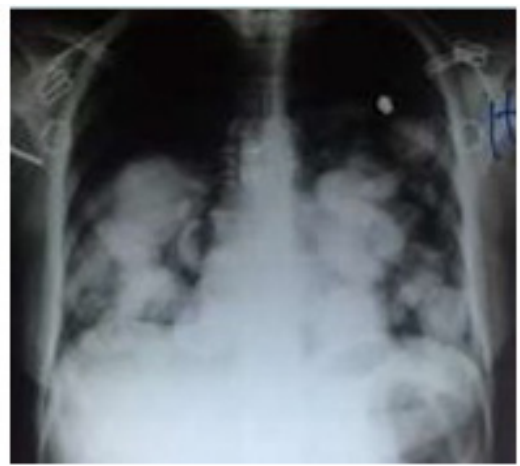

Figure 1: Chest X-ray showing multiple nodular opacities in both lungs resembling metastasis

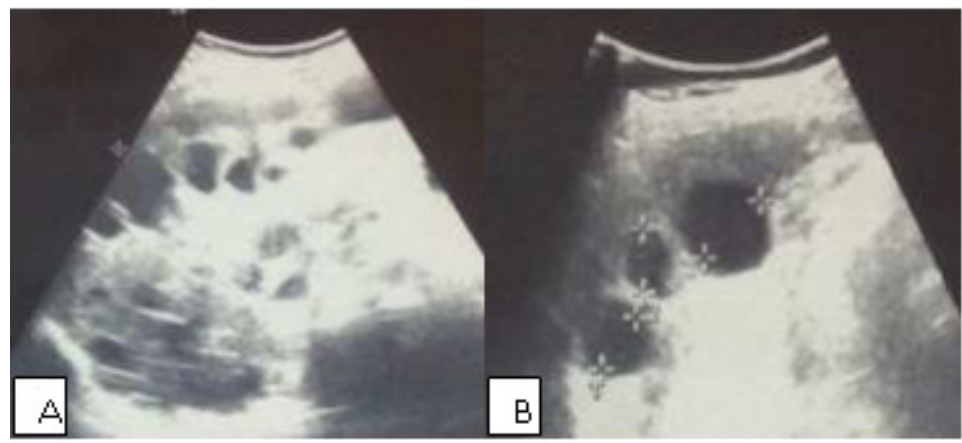

Figure 2: (A) Image from the liver ultrasound shows inummerable cysts of varyig sizes; and (B) Image from the spleen ultrasound demonstrate three cysts

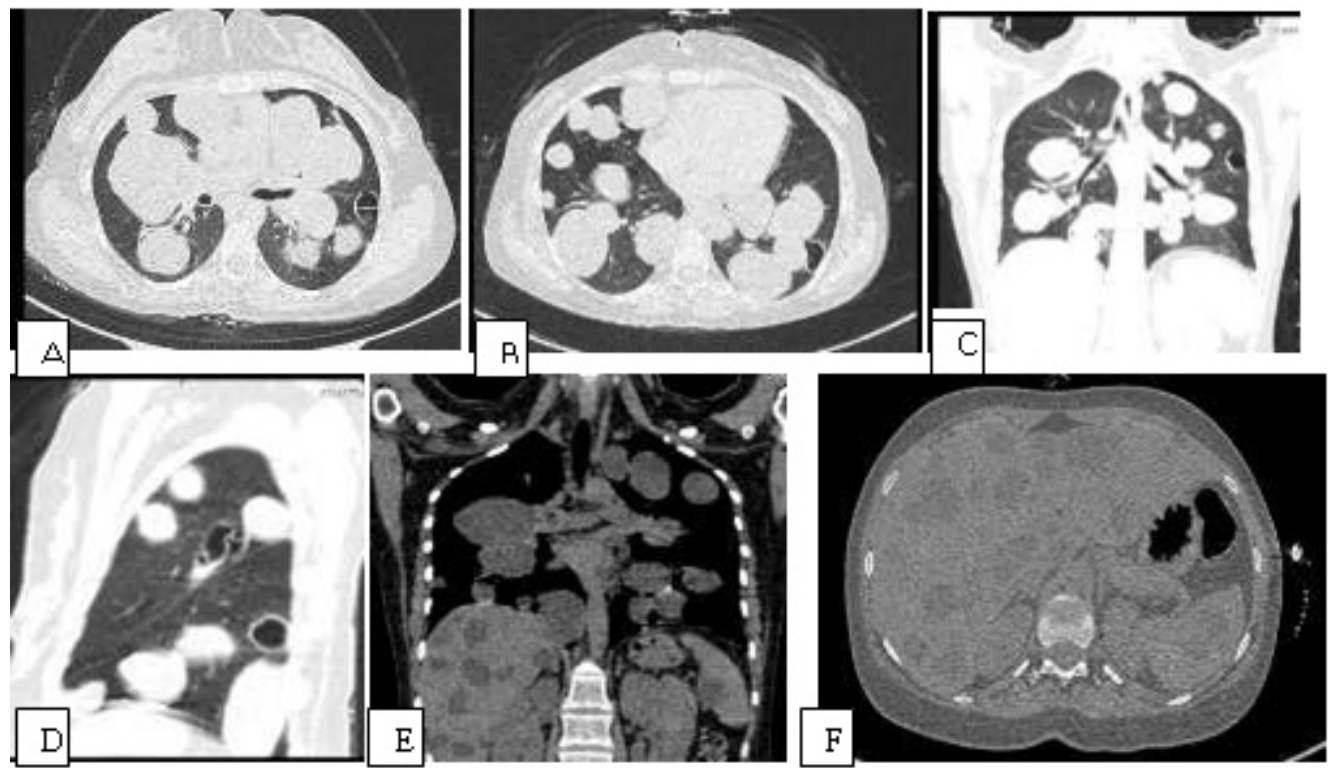

Figure 3: (A-D) Chest CT scan in lung window shows multiple lesions scattered through bilateral pulmonary parenchyma with some containing detached membranes indicating ruptured hydatids; (E,F) Chest and abdominal CT scan in soft tissue window shows a multiple cystic lesions in the lung, liver and spleen

\section{Discussion}

Hydatid disease is a parasitic infection caused by E. granulosus that represents a major public health problem in many countries including Middle East [4,5]. Its main hosts are dogs and foxes and its intermediate hosts are herbivores. Humans become infected 
by accidental ingestion of food contaminated with eggs of E. granulosus shed by definitive hosts [6]. The liver in adults and the lungs in children are the predominant sites. It develops outside these organs in $2.1 \%$ of the patients [7]. As reported in the literature, most primary infections in humans consist of a single cyst; however, 30\% of individuals have multiple cysts or multiple organ involvement $[2,8]$. Hydatid disease caused by E. granulosis is usually asymptomatic and diagnosed incidentally, because cyst growth rate is commonly slow and progressive. The most frequent symptoms include asthenia and abdominal pain. Patients may also present jaundice, hepatomegaly or anaphylaxis due to cyst leakage or rupture. Symptomatic hydatid disease of the lung, however, more often follows rupture of the cyst. The cyst may rupture spontaneously or as a result of trauma or secondary infection. If cysts in the lung rupture into the bronchi, intense cough may develop, followed by vomiting of hydatid material and cystic membranes as in this case. Simultaneous development of pulmonary and hepatic hydatid cysts is an unusual manifestation of hydatid disease that could be observed in less than $10 \%$ of cases [2]. It represents a specific entity called hepatopulmonary hydatidosis (HPH). HPH is more frequent in female patients over 40 years of age. The pulmonary cysts in HPH show a tendency to be bilateral and multiple [2]. The most common symptoms identified in patients with HPH are from the respiratory system, including cough, chest pain, dyspnea and hemoptysis. Secondary splenic hydatid disease usually occurs after systemic dissemination or intraperitoneal spread complicating ruptured hepatic hydatid cyst.

Herein, I report an exceptional and confusing presentation of hepatopulmonary and splenic hydatidosis due to E. granulosus that caused diagnostic challenge. The review of the literature revealed that there was only one other case characterized by a fulminant dissemination of hydatid cysts through organs reported in Tunisia [9].

The combined use of ultrasound and serological tests can facilitate the distinction of hydatid cysts from other hepatic cystic lesions [4]. Unilocular hydatid cyst appears on ultrasound as anechoic unilocular fluid-filled space with imperceptible walls and posterior acoustic enhancement. Ultrasound is not always able to differentiate hydatid cysts from other hepatic lesions, like tumors or liver abscesses. On CT scan and MRI, the simple hydatid cyst is defined as a well-demarcated water attenuation lesion that does not enhance after the administration of intravenous contrast [9]. Ultrasound may detect detached endocyst membranes and daughter cysts (vesicles within the mother cyst), which are highly specific for hydatid disease. Detachment of the membranes inside the cyst is referred to as the water lily sign. Among serologic tests, immune electrophoresis has been reported to be the most specific for the primary diagnosis and postsurgical follow-up and enzyme-linked immune sorbent assay (ELISA) represents a valuable diagnostic test for the initial screening [4]. In the present case, the serological tests were positive for E. granulosus by ndirect haemagglutination test. The main goal of the treatment of hepatic hydatid disease should be the complete elimination of the parasite and prevention of recurrence of the disease with minimum morbidity and mortality risks. Three therapeutic modalities are validated in the treatment of hydatid cysts: medical treatment, surgery (with open or laparoscopic approach) and percutaneous treatments. For many years, surgery was the first therapeutic option for hydatid disease [4]. Puncture, aspiration, injection, re-aspiration (PAIR) is a relatively recent and minimally invasive therapeutic option, introduced in the two past decades, and developed as an interesting alternative to surgery. It is a percutaneous treatment that consists of puncture of the cyst, aspiration of cyst fluid, injection of hypertonic saline and absolute alcohol that had a scolicidal effect, and re-aspiration of the cyst contents (PAIR) under sonographic guidance. This treatment is considered in case of single or multiple cysts, which cannot be operated [4]. PAIR represents an effective and safe procedure with low rates of complications and is the unique method that provides direct diagnosis concerning the parasitic nature of the cysts in doubtful cases [10]. Chemotherapy based on benz imidazole carbamate compounds (albendazole and mebendazole) have, for a long time, remained the cornerstone of medical therapy for echinococcosis [4]. It has very leffective results in patients with multiple or inoperable cysts and those with peritoneal hydatid cysts. Albendazole, an oral benzimidazole antihelmintic agent, is a drug of choice for the medical therapy of echinococcal disease [9]. The dose of drug is 10 to $15 \mathrm{mg} / \mathrm{kg}$ per day in 2 divided doses given in cycles of $4 \mathrm{wk}$, without drug therapy for $2 \mathrm{wk}$. This regimen should be maintained for several cycles depending on the severity of disease or the improvement of patients [9]. Praziquantel, an isoquinoline anthelmintic, is a potent protoscolicidal agent in vitro [9]. The usage of albendazole with praziquantel together may be more effective than albendazole alone. In the present case, the patient was treated with a comination of albendazole and praziquantel with a good general outcome.

\section{Conclusion}

I presented in this report a case of multi-organ hydatid disease with rapidly disseminated presentation which caused a diagnosis dilemma. The multiple lesions in chest X-ray can imitate the metastatic malignancy of lung. Ultrasound and CT findings in addition to serology tests results confirm the diagnosis. This case justifies that a high level of awareness towards the unusual presntations of parasitic infections of the lung and liver should be maintained especially in endemic countries.

\section{References}

1. Almalik A, Alsharidi A, Al-Sheef M, Enani M (2014) Disseminated abdominal hydatidosis: a rare presentation of common infectious disease. Case Rep Infect Dis $10.1155 / 2014 / 164787$.

2. Aribas OK, Kanat F, Turk E, Kalayci MU (2002) Comparison between pulmonary and hepatopulmonary hydatidosis. Eur J Cardiothorac Surg 21: 489-96.

3. Limeme M, Yahyaoui S, Zaghouani H, Ghannouchi M, Khnissi A, et al. (2014) Spontaneous intraperitoneal rupture of hepatic hydatid cyst: a rare cause of ascites. BMC Surg 14: 99.

4. Moro P, Schantz PM (2009) Echinococcosis: a review. Int J Infect Dis 13: 125-33.

5. Sheng Y, Gerber DA (2008) Complications and management of an echinococcal cyst of the liver. J Am Coll Surg 206: $1222-3$. 
6. McManus DP, Zhang W, Li J, Bartley PB (2003) Echinococcosis. Lancet 362: 1295-304.

7. Talaiezadeh AH, Maraghi S (2006) Hydatid disease in children: A different pattern than adults. Pak J Med Sci 22: 3329.

8. Karaoglanoglu N, Kurkcuoglu IC, Gorguner M, Eroglu A, Turkyılmaz A (2001) Giant hydatid lung cysts. Eur J Cardiothorac Surg 19 : $914-7$.

9. A Hammami, O Hellara, W Mnari, C Loussaief, F Bedioui, et al. (2015) Unusual presentation of severely disseminated and rapidly progressive hydatic cyst: Malignant hydatidosis. World J Hepatol 7: 633-7.

10. Siracusano A, Teggi A, Ortona E (2009) Human cystic echinococcosis: old problems and newperspectives. Interdiscip Perspect Infect Dis $2009: 474368$. 